\title{
ANALISIS OF THE NONLINEAR DISTRIBUTED CONTROL SYSTEM'S SUSTAINABILITY
}

\author{
Alexander B. Chernyshev, Karina V. Martirosyan and \\ Alexander V. Martirosyan \\ North-Caucasian Federal University, Branch in Pyatigorsk, Russian Federation, \\ 357500, Pyatigorsk, Krasnoarmeyskaya Str., 20, Russia
}

Received 2014-06-19; Revised 2014-07-09; Accepted 2014-07-12

\begin{abstract}
Systems' stability is the topical issue of control systems' development province. In this study the research of methodology of the nonlinear distributed control systems' stability assessment is conducted. Research of the standard distributed links' frequency surfaces is executed, limit characteristics of the parameters influencing by sight of spatial hodograph are investigated. The purpose of the work is to present the new method of systems' stability assessment and to the results of its using. The offered method of the absolute stability analysis for a class of systems with the distributed parameters allows to investigate the dynamic characteristics of the nonlinear distributed control systems. The specified method is received on the basis of frequency methods and representation of straight line's slope limiting sector of the nonlinear characteristic in the form of a spatial and amplified-extensive section. On the basis of the modeling results studying the general method of stability assessment, which can be used for the models of nonlinear control systems considered in work is offered. The criterion of nonlinear distributed control systems' absolute stability with the nonlinear characteristic independent of object's spatial coordinates is formulated and described. Results of this method's application for the analysis of the nonlinear distributed systems' stability which proves the method's assurance are considered.
\end{abstract}

Keywords: Control Systems Modeling, Nonlinear Distributed Control Systems, Control System's Stability, Isochronous Surface of Nonlinear Control Systems

\section{INTRODUCTION}

For the first time the concept "the distributed system" was introduced by the doctor of engineering A.G. Butkovsky who is the recognized scientist in the field of distributed systems' management. Researches in this direction were continued by professor E.Ya. Rapoport and T.K. Sirazetdinov, whose fundamental works became the beginning of theory's of systems' with the distributed parameters management development (Rapoport, 2005). The significant contribution to research of this type systems was made by the French mathematician Jacques-Louis Lions who is the one of the leading scientists in the field of the differential Equation in private derivatives theory and stochastic management (Lions, 1973).

The research problem of nonlinear control systems' absolute stability arises because of the instability of nonlinear characteristic and can be characterizes only by certain area (Malkov and Pershin, 2007). Rather wide class of nonlinear control systems is represented by the systems which block diagram is represented by consecutive connection of the nonlinear block and linear part (Lions, 1988). In this case it is possible to use the tool of linear part's of system transfer functions (Sergienko and Deineka, 2005).

The majority of received results in the theory of the systems with distributed parameters are treat linear Corresponding Author: Alexander B. Chernyshev, North-Caucasian Federal University, Branch in Pyatigorsk, Russian Federation, 357500, Pyatigorsk, Krasnoarmeyskaya str., 20, Russia 
systems. Real systems of automatic control are not purely linear and in some cases their behavior can't even be approximate is described by the linear differential Equation (Miller and Michel, 2007). Research of nonlinear systems is connected with overcoming of considerable mathematical difficulties since there is no uniform exact method of the nonlinear Equations' solution and at research of various nonlinear systems it is necessary to find special private methods (Martirosyan and Martirosyan, 2014). Research of the distributed control systems has important applied value, for example, for development of management systems for hydromineral resources (Martirosyan, et al., 2013).

Representation of discrete managing directors of influences in the form of delta functions allows to investigate a class of systems with the distributed parameters for which there is a fundamental decision in the form of decomposition on own vector functions of the object's operator (Finkbeiner and Peter, 2012). Research of operating impacts' sampling parameters' influencing on process of regulation allows to carry out regulation of nonlinear discrete systems in a relay mode. Representation of the straight line's slope, that limits the sector of nonlinear characteristic, in the form amplifiedextensive section gain, allows to formulate the frequency criterion of absolute stability adapted for a class of systems with the distributed parameters.

\section{MATERIALS AND METHODS}

Let the nonlinear element be set by function $z=$ $\varphi(\sigma)$ which puts value $z(x, y, t)$ like an output signal of a link to value $\sigma(x, y, t)$ of an entrance signal in compliance, i.e., $z(x, y, t)=\varphi(\sigma(x, y, t))$.

Let the image according to Laplace be set under zero initial conditions of input action $\sigma(x, y, s)$. Input action can be presented in the form of Fourier's number Equation 1 and 2:

$$
\sigma(x, y, s)=\sum_{\eta, \gamma=1}^{¥} \sum_{\xi=1}^{4} C_{\eta, \gamma, \xi}(s) \times B_{\eta, \gamma, \xi}(x, y)
$$

Where:

$$
\psi_{n}=\frac{\pi \eta}{l_{x}} ; \tilde{\psi}_{\gamma}=\frac{\pi \gamma}{l_{y}}
$$

Let's consider a nonlinear system with steady linear part. Assume, that nonlinear element has the unambiguous static characteristic. It is known, that for systems with the concentrated parameters the static characteristic meets conditions Equation 3:

$$
0 \leq \varphi(\sigma) \leq k \sigma ; \varphi(0)=0
$$

i.e., the characteristic of a nonlinear element must belong to sector $[0 ; k]$. It is the main sign of a considered class of curvilinearity.

In relation to the systems with the distributed parameters, we will consider the coefficient $k$ as coefficient of amplified-extensive section gain (Fig. 1) Equation 4:

$$
K(G)=E_{1}\left[\frac{n_{1}-1}{n_{1}}+\frac{1}{n_{1}} G\right], 0 \leq G \leq \infty
$$

Then the straight-line Equation, limiting sector of nonlinearity from above, for each contour it is possible to write down in a look Equation 5:

$$
z_{n}=E_{1}\left[\frac{n_{1}-1}{n_{1}}+\frac{1}{n_{1}} G\right] \cdot \sigma_{n}
$$

where, $n$-contour number. Then the Equation of the surface limiting area of nonlinearity, expressed through the private derivatives, can be written down in a look Equation 6:

$$
\begin{aligned}
& z_{n}=E_{1}\left[\frac{n_{1}-1}{n_{1}}+\frac{1}{n_{1}} \nabla^{2}\right] \\
& \sigma=E_{1}\left[\frac{n_{1}-1}{n_{1}}+\frac{1}{n_{1}}\left(\frac{\partial^{2}}{d x^{2}}+\frac{\partial^{2}}{d y^{2}}\right)\right] \\
& \sigma(x, y, t)=E_{1}\left[\begin{array}{l}
\frac{n_{1}-1}{n_{1}} \sigma(x, y, t)- \\
\frac{1}{n_{1}}\left(\frac{\partial^{2} \sigma(x, y, t)}{d x^{2}}+\frac{\partial^{2} \sigma(x, y, t)}{d y^{2}}\right)
\end{array}\right]
\end{aligned}
$$

From where we will receive that, if nonlinear entrance influence doesn't depend on spatial coordinates $x, y$, expression will be as follows Equation 7 and 8:

$$
z=E_{1} \frac{n_{1}-1}{n_{1}} \sigma(t)
$$

Where:

$$
k=E_{1} \frac{n_{1}-1}{n_{1}}
$$




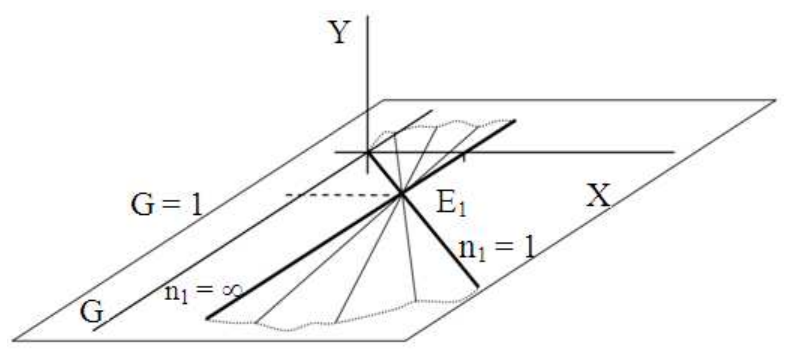

Fig. 1. Hodograph of the amplified-extensive section

That is, the straight-line Equation $z=k \cdot \sigma(t)$ doesn't depend on value of the generalized coordinate $G$. Value of slope $k$ depends on the set gain $E_{1}$ and on the weight coefficient $n_{1}$, which selection will allow to minimize sector, which possesses the nonlinear characteristic (Chernyshev and Ilushin, 2010).

If input action is set in the form of the image according to Laplace $\sigma(x, y, s)$, the surface, limiting sector $[0, k]$ from above will have an appearance Equation 9 and 10:

$z=E_{1}\left[\frac{n_{1}-1}{n_{1}}+\frac{1}{n_{1}} G\right] \cdot \sigma(x, y, s)$

Where:

$$
k=E_{1}\left[\frac{n_{1}-1}{n_{1}}+\frac{1}{n_{1}} G\right]
$$

\section{RESULTS}

V. M. Popov offered frequency criterion of absolute stability determination for the nonlinear systems with the concentrated parameters, That is the system's stability at any initial deviations for any form of the nonlinear characteristic belonging to some certain class (Chernyshev, 2010). Rather wide class of nonlinear control systems is represented by the systems which block diagram is submitted consecutive connection of the nonlinear block and linear part. In this case it is possible to use the device of transfer functions of the system's linear part.

Let's formulate the generalized criterion of nonlinear distributed control systems absolute stability.

Let conditions be satisfied:

- All poles of the system's linear part's transfer function have negative valid parts (i.e., the linear part of the opened system is steady)
- The characteristic of a nonlinear element $z=$ $\varphi(\sigma(x, y, t))$ must belong to an area that is limited by plane $z=0$ and a surface Equation 11 and 12:

$$
z=E_{1}\left[\begin{array}{l}
\frac{n_{1}-1}{n_{1}} \sigma(x, y, t)- \\
\frac{1}{n_{1}}\left(\frac{\partial^{2} \sigma(x, y, t)}{d x^{2}}+\frac{\partial^{2} \sigma(x, y, t)}{d y^{2}}\right)
\end{array}\right]
$$

$$
\text { i.e., }
$$

$$
\begin{aligned}
& \phi(0)=0,0 \leq \frac{\phi(\sigma(x, y, t))}{\sigma(x, y, t)} \\
& \leq E_{1}\left[\frac{n_{1}-1}{n_{1}}-\frac{1}{n_{1}}\left(\frac{\partial^{2}}{d x^{2}}+\frac{\partial^{2}}{d y^{2}}\right)\right]
\end{aligned}
$$

At all $\sigma(x, y, t) \neq 0$.

If input action is set in the form of the image according to Laplace $\sigma(x, y, s)$, the surface limiting area from above will have an appearance Equation 13 and 14:

$z=E_{1}\left[\frac{n_{1}-1}{n_{1}}+\frac{1}{n_{1}} G\right] \cdot \sigma(x, y, s)$

$\phi(0)=0,0 \leq \frac{\phi(\sigma(x, y, s))}{\sigma(x, y, s)} \leq E_{1}\left[\frac{n_{1}-1}{n_{1}}-\frac{1}{n_{1}} G\right]$

At all, $\sigma(x, y, s) \neq 0$, where Equation 15:

$G=\left(\frac{n \pi}{l_{x}}\right)^{2}+\left(\frac{n \pi}{l_{y}}\right)^{2}=\frac{n^{2} \pi^{2}\left(l_{x}^{2}+l_{y}^{2}\right)}{l_{x}^{2} l_{y}^{2}}$

There is a real number $q$ such that at all $\omega \in[0 ; \infty]$ the inequality is carried out Equation 16:

$$
\operatorname{Re}[(1+j \omega q) W(j \omega)]>-\frac{1}{E_{1}\left[\frac{n_{1}-1}{n_{1}}-\frac{1}{n_{1}} G\right]}
$$

Then at any limited initial deviations from zero value, function $\sigma(x, y, t)$ remains limited at $t>0$ and $\sigma(x, y, t) \rightarrow 0$, at $t \rightarrow \infty$, i.e., the system will be asymptotically steady as from limitation $\sigma(x, y, t)$ follows $Q(x, y, t)$ limitation and from aspiration of $\sigma(x, y, t)$ to zero follows that $Q(x, y, t) \rightarrow 0$ at $t \rightarrow \infty$. 


\section{DISCUSSION}

The realization of input action in systems with the distributed parameters is enabled by its sampling on spatial coordinates. Research of the nonlinear systems' regulator's absolute stability with the distributed parameters on the basis of frequency methods is conducted on the basis of straight line's slope representation of the nonlinear characteristic's limiting sector in the form of a spatial and intensifying link.

Thus, it is possible to give the following graphic interpretation of the generalized criterion of Popov (Chernyshev, 2010).

If transfer function of the open-loop system has no the poles, lying in the right half-plane, then for absolute stability of the closed-loop system it is enough (Liao and $\mathrm{Yu}, 2008)$ that the modified spatial hodograph didn't cross the surface passing through the line $\left\{\operatorname{Re}(W)=-\frac{1}{E_{1}\left[\frac{n_{1}-1}{n_{1}}-\frac{1}{n_{1}} G\right]} ; \operatorname{Im}(W)=0\right\}$ and a straight line $\{\operatorname{Re}(W)=0 ; \operatorname{Im}(W)=q ; G\}$.

As shown in Fig. 2, the surface consisting of Popov's straight lines, aspires to an axis $G$ in a limit, at the same time the hodograph's module decreases.

Thus, the surface as though catches up with a hodograph. If they are crossed, at least in harmonicas of a big order, the system becomes unstable.

Using the modification of nonlinear systems' with the distributed parameters absolute stability criterion, the analysis method for practical use can be offered.

To choose some final number of values of $G_{i}, i=\overline{1, m}$ Equation 17:

$$
\begin{aligned}
& G=\left(\frac{\pi}{l_{x}}\right)^{2}+\left(\frac{\pi}{l_{y}}\right)^{2}=\frac{\pi^{2}\left(l_{x}^{2}+l_{y}^{2}\right)}{l_{x}^{2} l_{y}^{2}}, \\
& G=\left(\frac{2 \pi}{l_{x}}\right)^{2}+\left(\frac{2 \pi}{l_{y}}\right)^{2}=\frac{4 \pi^{2}\left(l_{x}^{2}+l_{y}^{2}\right)}{l_{x}^{2} l_{y}^{2}}, \\
& \ldots, \\
& G_{m}=\left(\frac{m \pi}{l_{x}}\right)^{2}+\left(\frac{m \pi}{l_{y}}\right)^{2}=\frac{m^{2} \pi^{2}\left(l_{x}^{2}+l_{y}^{2}\right)}{l_{x}^{2} l_{y}^{2}}
\end{aligned}
$$

To construct the schedule of the nonlinear characteristic $z=\phi(\sigma)$ and to carry out a tangent to the schedule, passing through the beginning of coordinates (Damm and Finkbeiner, 2014).

To determine the tangent slope $k$ Equation 18:

$z=\frac{z_{1}}{\sigma_{1}} \sigma ; k=\frac{z_{1}}{\sigma_{1}}$

From a ratio Equation 19:

$k=E_{1}\left[\frac{n_{1}-1}{n_{1}}+\frac{1}{n_{1}} G\right]$

At concrete values $G_{i}, i=\overline{1, m}$ values of slopes will be as follows Equation 20:

$$
\begin{aligned}
& k=E_{11}\left[\frac{n_{11}-1}{n_{11}}+\frac{1}{n_{11}} G_{1}\right] ; \\
& k=E_{12}\left[\frac{n_{12}-1}{n_{12}}+\frac{1}{n_{12}} G_{2}\right] ; \\
& \ldots ; \\
& k=E_{1 m}\left[\frac{n_{1 m}-1}{n_{1 m}}+\frac{1}{n_{1 m}} G_{m}\right]
\end{aligned}
$$

To pick up coefficients $n_{1}$ and $E_{1}$ so, that the nonlinear characteristic got to curvilinearity sector for each value $G_{i}, i=\overline{1, m}$. For example, at $n_{1}=1$, we receive $k=E_{1} G$, then $E_{1}=\frac{k}{G}$ (Chernyshev, 2009).

Taking into account inequalities $G_{1}<G_{2}<\ldots<G_{m}$, the greatest of values $E_{1 i}, i=\overline{1, m}$ are getting out, i.e., $E_{1}=\frac{k}{G_{1}}$ or $E_{1}=\frac{k l_{x}^{2} l_{y}^{2}}{\pi^{2}\left(l_{x}^{2}+l_{y}^{2}\right)}$.

For each of values $G_{i}, i=\overline{1, m}$. The modified hodograph of transfer function of the system's linear part $W_{i}\left(G_{i}, s\right), . i=\overline{1, m}$ must be constructed and the points are defined on axes $X=\operatorname{Re}\left(W_{i}\right)$ equal Equation 21:

$-\frac{1}{E_{l}\left[\frac{n_{l}-1}{n_{l}}+\frac{1}{n_{l}} G_{i}\right]}$

For example, for values Equation 22:

$n_{1}=1, E_{1}=\frac{k l_{x}^{2} l_{y}^{2}}{\pi^{2}\left(l_{x}^{2}+l_{y}^{2}\right)}$ 


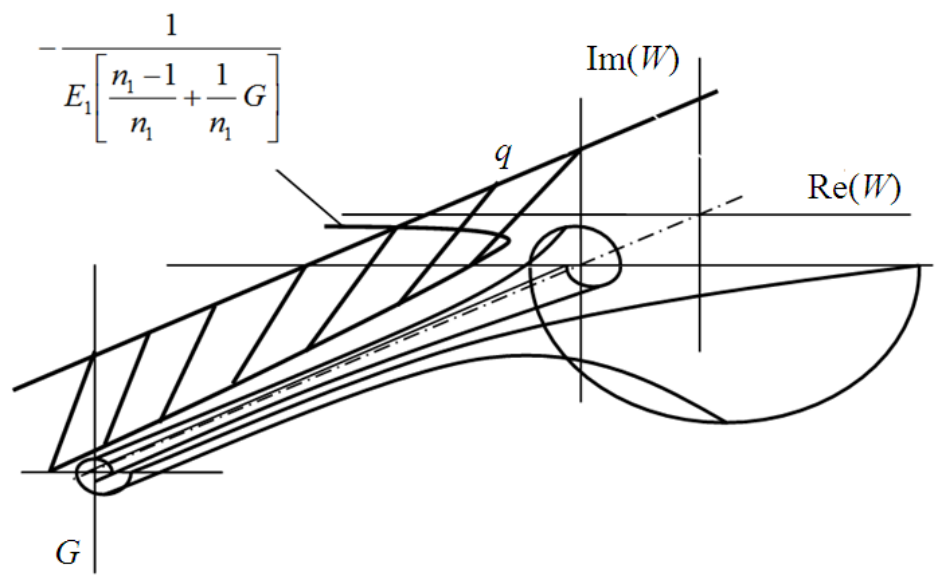

Fig. 2. Graphic interpretation of the distributed control system's absolute stability analysis

We will receive Equation 23 and 24:

$$
X_{i}=-\frac{1}{E_{1} G_{i}}
$$

$$
\text { i.e., }
$$

$X_{1}=-\frac{1}{k}, X_{2}=-\frac{1}{4 k}, \ldots, X_{m}=-\frac{1}{m^{2} k}$

7. If for each point of $X_{i}, i=\overline{1, m}$ on an axis $Y=$ $\omega \operatorname{Im}\left(W_{i}\right)$ the point $Y_{i}=q$, such that the straight line drawn through these points doesn't cross the modified hodograph would be found out, the nonlinear system is absolutely steady. The offered method of the absolute stability analysis for a class of the systems with the distributed parameters allows to investigate dynamic characteristics of the nonlinear distributed control systems (Martirosyan and Martirosyan, 2013).

\section{CONCLUSION}

The offered method of the absolute stability analysis for a class of the systems with the distributed parameters allows to investigate dynamic characteristics of the nonlinear distributed control systems. The analysis of spatial hodograph of the standard distributed links allows to build the difficult nonlinear distributed control systems' hodograph of linear part that gives the chance of researching of their absolute stability with the application of frequency criterion. On the basis of theoretical development the practical methods, allowing to make the analysis and synthesis of the automatic control systems are received. The developed methods allow to choose technological parameters of adjustable processes, proceeding from the demanded accuracy of regulation. Using of these parameters will provide a steady mode of functioning of objects at nonlinear influences. The absolute stability criterion for the nonlinear distributed control systems is formulated. Graphic interpretation of the generalized Popov's criterion of stability is also given. The only problem of the research is that this method works only with homogeneous boundary conditions. The future plan is to improve the method to make its using with different boundary conditions possible.

\section{ACKNOWLEDGMENT}

The researchers acknowledge receiving support from the North-Caucasus Federal University represented by Dr. Ivan Pershin and Dr. Tatyana Shebzukhova. We are responsible for all errors as well as heavy style of the manuscript.

\section{REFERENCES}

Chernyshev, A.B. and U.V. Ilushin, 2010. Stability of the distributed systems with discrete managing directors of influences. Proc. News Izvestiya: South Federal Uni., 12: 166-171.

Chernyshev, A.B., 2009. Adaptation of distributed parameters system's frequency criterion of absolute stability. Mechatron, Automat. Manage., 7: 13-18. 
Chernyshev, A.B., 2010. Research of the nonlinear distributed systems' absolute stability. Automat. Modern Technol., 4: 21-26.

Damm, W. and B. Finkbeiner, 2014. Automatic Compositional Synthesis of Distributed Systems. In: FM 2014: Formal Methods, Jones, C., P. Pihlajasaari and J. Sun (Eds.), Springer International Publishing, ISBN-10: 978-3-319-06409-3, pp: 179193.

Finkbeiner, B. and H.J. Peter, 2012. Template-based Controller Synthesis for Timed Systems. In: Tools and Algorithms for the Construction and Analysis of Systems, Flanagan, C. and B. König (Eds.), Springer Berlin Heidelberg, ISBN: 978-3-642-28755-8, pp: 392-406.

Liao, X. and P. Yu, 2008. Absolute Stability of Nonlinear Control Systems. 1st Edn., Illustrated, Springer Science and Business Media, New York, ISBN-10: 140208482X, pp: 396.

Lions, J.L., 1973. The optimal control of distributed systems. Adv. Math. Sci., 28: 15-46.

Lions, J.L., 1988. Exact controllability, stabilization and perturbations for distributed systems. SIAM Rev., 30: 1-68. DOI: 10.1137/1030001

Malkov, A.V. and I.M. Pershin, 2007. Distributed Controller Synthesis for the Hydrolitosphere Processes Management Systems. 1st Edn., Moscow, Science World, pp: 235-240.
Martirosyan, A.V. and K.V. Martirosyan, 2013. Modeling of information system "Caucasus Mineral Water's hydromineral resources". Proceedings of the 4th International Scientific and Practical Conference "Science and Society, (CSS' 13), pp: 158-165.

Martirosyan, A.V. and K.V. Martirosyan, 2014. Ensuring sustainable model of mineral water deposits operating modes on the example of the deposit Nagutskaya. Int. Sci. Technical J., 6: 89-93.

Martirosyan, K.V., A.V. Martirosyan and T.S. Kapylova, 2013. The model of mineral water deposits sustainable management using the decision support system. World Applied Sci. J., 27: 101-106. DOI: 10.5829/idosi.wasj.2013.27.01.13599

Miller, R.K. and A.N. Michel, 2007. Ordinary Differential Equations. 1st Edn., Dover Publications, USA, pp: 250.

Rapoport, E.J., 2005. Analysis and Synthesis of the Automatic Control Systems with Distributed Parameters. 1st Edn., Higher School, Moscow, pp: 292.

Sergienko, I.V. and V. S. Deineka, 2005. Optimal Control of Distributed Systems with Conjugation Conditions. 1st Edn., Kluwer Academic Publishers, USA, pp: 133-145. 